\title{
Progression and prognosis in pure autonomic failure (PAF): comparison with multiple system atrophy
}

\author{
N Mabuchi, M Hirayama, Y Koike, H Watanabe, H Ito, R Kobayashi, K Hamada, G Sobue
}

J Neurol Neurosurg Psychiatry 2005;76:947-952. doi: 10.1136/jnnp.2004.049023

See end of article for authors' affiliations

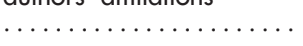

Correspondence to: Dr Gen Sobue,

Department of Neurology,

Nagoya University

Graduate School of

Medicine, Nagoya 466-

8550, Japan; sobueg@

med.nagoya-u.ac.jp

Received 6 July 2004

In revised form

15 October 2004

Accepted 15 October 2004
Objective: To clarify the progression of autonomic symptoms and functional deterioration in pure autonomic failure (PAF), particularly in comparison with multiple system atrophy (MSA).

Methods: The investigation involved eight patients with $P A F(M / F=7 / 1$; mean age at onset, 57 years) and 22 with probable MSA matched for age at onset ( $M / F=14 / 8$; onset 56 years). Subjects were followed up for neurological symptoms, activities of daily living, and autonomic function for more than seven years. Autonomic functional tests were carried out.

Results: In PAF, fainting or sudomotor dysfunction occurred first, followed by constipation and syncope. Urinary dysfunction developed late, and respiratory dysfunction was not evident. This clinical course contrasted sharply with that in MSA, where early urinary dysfunction usually proceeded to sudomotor dysfunction or orthostatic hypotension $(p=0.004)$, followed by respiratory dysfunction $(p=0.0004)$. Results of pharmacological tests also distinguished PAF from MSA. Progression and prognosis in patients with PAF did not worsen, unlike the steady progressive autonomic dysfunction in MSA $(p<0.0001$, $p<0.0001, p=0.0009$, and $p=0.003$, for progression to modified Rankin scale grade III, IV, V, and death, respectively).

Conclusions: The time course and pattern of progression of autonomic failure differed significantly between PAF and MSA. Patients with PAF had slower functional deterioration and a better prognosis.
$\mathrm{P}$ ure autonomic failure (PAF) is a sporadic idiopathic neurodegenerative disorder characterised by gradually progressive severe autonomic disturbances without other neurological features. In the past, PAF was defined as severe orthostatic hypotension without other neurological deficits, and was referred to as idiopathic orthostatic hypotension. However, this has proved to be a heterogeneous condition, including diseases such as PAF, acute autonomic neuropathy, the early stages of Shy-Drager syndrome, and Parkinson's disease with autonomic failure. ${ }^{1-6}$

Bannister et $\mathrm{al}^{7}$ classified primary autonomic failure into three categories: Parkinson's disease with autonomic failure, multiple system atrophy (MSA), and pure autonomic failure. In 1996, a consensus statement was established concerning $\mathrm{PAF}^{8}{ }^{8}$ but it has remained uncertain whether the autonomic failure of PAF can readily be distinguished from those of MSA and Parkinson's disease with autonomic failure. In addition, although the clinical course of both MSA and Parkinson's disease with autonomic failure has been described to some extent, details of the natural history of PAF have not been fully assessed because of its rarity and very slow progression. ${ }^{9-11}$ Previous reports have noted longer survival in patients with PAF than in those with MSA. ${ }^{32-15}$ Orthostatic hypotension and anhidrosis/hypohidrosis are the main clinical symptoms in PAF, but their severity, prognosis, and progression have been only incompletely assessed. To clarify the clinical features, particularly the natural course of PAF, we observed eight patients who fulfilled the PAF consensus statement and maintained a follow up for at least five years. We show that their features are distinct from those of another form of primary autonomic failure, MSA.

\section{METHODS}

\section{Patients}

We examined eight patients with PAF (seven men, one woman; mean (SD) age at onset, 57 (14) years; mean age at first evaluation, 68 (12) years; mean duration from onset to most recent evaluation, 19 (10) years) who were referred to the Nagoya University Hospital or its affiliated hospitals in Aichi prefecture between 1988 and 1997. We evaluated these patients clinically from onset for between seven and 32 years. We reviewed the clinical records preceding our own follow up period, and also obtained information by interviewing the patients and family members.

According to the consensus statement, ${ }^{8}$ PAF is characterised by orthostatic hypotension, various other autonomic signs without more widespread neurological involvement, and a low resting supine plasma noradrenaline concentration. The statement acknowledged that some patients would later prove to have other disorders such as $\mathrm{MSA}^{8}$ but did not state how long a period of follow up was required to confirm a diagnosis of PAF. Early MSA with predominant autonomic failure is particularly difficult to distinguish from PAF. We estimated that most MSA patients can be diagnosed by follow up for five years or more after onset, ${ }^{816}$ and we therefore serially examined putative PAF patients for more than five years from onset to exclude those with MSA. We also excluded patients with acute autonomic neuropathy, Parkinson's disease with autonomic failure, and other diseases presenting with autonomic signs by neurological examination, imaging (magnetic resonance imaging and positron emission tomography), and neurophysiological tests.

We also investigated 22 probable MSA patients ${ }^{17}$ matched according to age at onset ( 14 men, eight women; mean age at onset, 56 (8) years; mean age at first autonomic test, 61 (7) years; mean interval from onset, 8 (3) years) who had detailed clinical information particularly concerning autonomic features, and follow up intervals from over five years to 16 years after onset. All patients with MSA presented with autonomic failure as an initial symptom or with predominant autonomic failure at their first clinical visit, and fulfilled the criteria for a probable MSA diagnosis. ${ }^{17}$

Abbreviations: AVP, arginine-vasopressin; HUT, head up tilt test; MSA, multiple system atrophy; PAF, pure autonomic failure 
Table 1 Clinical profiles of eight patients with pure autonomic failure at their first visit

\begin{tabular}{|c|c|c|c|c|c|c|c|c|}
\hline \multirow[b]{2}{*}{ Variable } & \multicolumn{8}{|c|}{ Patient } \\
\hline & 1 & 2 & 3 & 4 & 5 & 6 & 7 & 8 \\
\hline Sex & $M$ & $M$ & $M$ & $M$ & $M$ & $M$ & $M$ & $\mathrm{~F}$ \\
\hline Onset age (y) & 35 & 68 & 72 & 78 & 50 & 52 & 51 & 50 \\
\hline Time until first evaluation $(y)$ & 17 & 1 & 10 & 5 & 27 & 7 & 5 & 13 \\
\hline Duration of observation (y) & 32 & 7 & 12 & 12 & 32 & 14 & 15 & 29 \\
\hline Hypohidrosis & + & + & + & + & + & + & + & + \\
\hline Faintness & + & + & + & + & + & + & + & + \\
\hline Syncope & - & - & - & - & + & + & + & + \\
\hline Constipation & + & - & - & - & + & - & + & - \\
\hline Difficulty in urination & - & - & + & - & + & - & - & - \\
\hline Incontinence/urinary urgency & - & - & - & - & - & + & + & + \\
\hline Respiratory disturbance & - & - & - & - & - & - & - & - \\
\hline Plasma noradrenaline $(\mathrm{pg} / \mathrm{ml})$ * & 30 & 43 & 25 & 83 & 50 & 34 & 14 & 10 \\
\hline Orthostatic hypotension & + & + & + & + & + & + & + & + \\
\hline Denervation supersensitivity & + & + & + & + & + & + & + & + \\
\hline Modified Rankin scale & 0 & 0 & 0 & 0 & 0 & 0 & 0 & 0 \\
\hline
\end{tabular}

\section{Procedures}

We evaluated all eight patients with PAF and 22 with MSA with a passive multistage head up tilt test (HUT) and a noradrenaline infusion test. The HUT was performed as follows. Blood pressure and heart rate were measured continuously by tonometry (SA-250; Colin, Komaki, Japan). After blood pressure stabilised at the supine stage, changes in blood pressure and heart rate were recorded continuously through $20^{\circ}, 40^{\circ}$, and $60^{\circ}$ head up tilting for five minutes each. Orthostatic hypotension was defined as a fall in systolic blood pressure of more than $30 \mathrm{~mm} \mathrm{Hg}$ during the $60^{\circ}$ head up tilt. ${ }^{18}$

Blood samples were collected at the rested supine stage and after $60^{\circ}$ head up tilting from all patients for evaluation of plasma noradrenaline and arginine-vasopressin (AVP). Differences in AVP between after $60^{\circ}$ head up tilting and the supine position were calculated as $\triangle$ AVP. Additionally, a noradrenaline infusion test was carried out as follows. A very low $(0.3 \mu \mathrm{g} / \mathrm{min})$ or a low $(3 \mu \mathrm{g} / \mathrm{min})$ concentration of noradrenaline was infused intravenously while blood pressure was monitored for changes. If diastolic or systolic blood pressure rose by more than $10 \mathrm{~mm} \mathrm{Hg}$ or $25 \mathrm{~mm} \mathrm{Hg}$, respectively, the patient was considered to have denervation supersensitivity involving the sympathetic nervous system. ${ }^{19}$ Four patients were re-evaluated two, five, six, and 11 years later, respectively. We also carried out ${ }^{123}$ I-metaiodebenzylguanidine (MIBG) scintigraphy and evaluated the heart/ mediastinum (H/M) ratio from delayed images, as previously described..$^{20-22}$

We followed up all eight patients and noted the time points when new autonomic symptoms appeared, including hypohidrosis, faintness and syncope, constipation, urinary dysfunction, impotence, and respiratory distress, and considered such clinical features in sequence to assess the natural clinical course. We evaluated hypohidrosis in terms of inspection of the skin and recording of patient symptoms. Dry skin or reduced perspiration was noted on some parts of the body, with compensatory hyperhidrosis elsewhere. Patients often noted their reduced perspiration in summer and felt severe fatigue, which sometimes limited their capacity for outdoor work. Faintness was defined as a floating sensation while in the upright position without loss of consciousness, or as symptomatic orthostatic hypotension during the head up tilt test. Syncope was defined as a blackout or loss of consciousness, including severe blurred vision. Constipation was defined by the passage of stools at intervals of three days or more, or complaints of straining.
Urinary dysfunction was defined as urination twice at night or more than five times in the daytime, urinary urgency, incontinence, or difficulty in urination. Impotence was defined as difficulty in achieving normal sexual function. Respiratory disturbances were defined either as the presence of sleep apnoea, including heavy snoring, or as difficulty in respiration. Onset of an autonomic symptom was defined as the time when the patient first noted the symptom.

\section{Statistics}

The Mann-Whitney U test for non-parametric statistics was used as appropriate._Kaplan-Meier analyses were employed to estimate the natural course of autonomic features and disease progression, assessed by the modified Rankin scale in both PAF and MSA patients. Log-rank test statistics were used to determine whether the Kaplan-Meier curves differed between PAF and MSA. Calculations were done using the statistical software package Stat View (Abacus Concepts, Berkeley, California, USA). Statistical significance was defined as a probability ( $p$ ) value of $<0.05$.

\section{RESULTS}

Clinical profiles of PAF on the first visit to the hospitals Clinical profiles of the eight patients with PAF at their first examination at our hospital are presented in table 1. They had many complaints suggesting autonomic disturbances, but the specific features varied. The earliest age at onset was 35 years, and the latest was 78 years. The interval from onset to presentation at our hospital varied from one to 27 years. Each patient showed various autonomic disturbances at that time, but faintness and hypohidrosis had been experienced by all patients. Other autonomic symptoms were as follows: urinary dysfunction in five, syncope in four, constipation in three, and impotence in two. All patients had very low plasma noradrenaline concentrations, orthostatic hypotension, and denervation supersensitivity according to the noradrenaline infusion test.

\section{Clinical manifestations of MSA}

The initial symptoms in all 22 patients with MSA were those of autonomic failure. Median time from onset to the presence of concomitant autonomic and motor manifestations (evolution from onset to probable MSA) was 2.0 years (range 1 to 10). At the first clinical visit, seven of the 22 patients presented with severe autonomic failure but failed to fulfil consensus diagnostic criteria of MSA. 

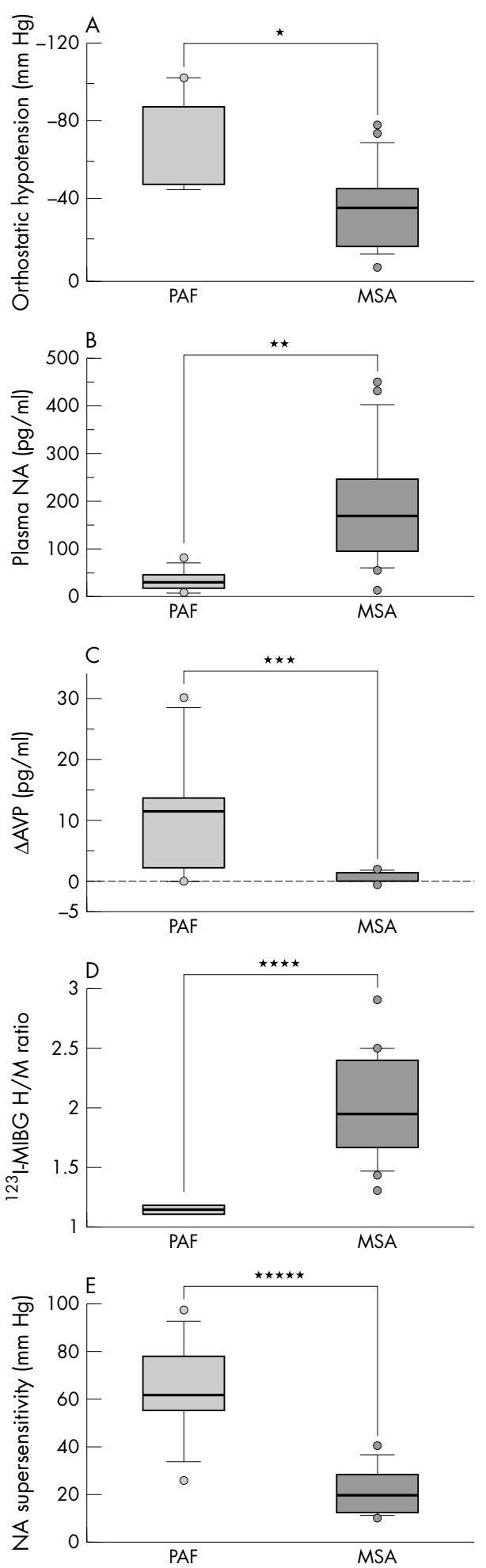

Figure 1 Box and whisker plot of the autonomic nervous testing comparing pure autonomic failure (PAF) with multiple system atrophy (MSA). (A) Systolic blood pressure fall during orthostatic hypotension. (B) Plasma noradrenaline (NA) concentration. (C) Differences in arginine-vasopressin (AVP) concentration between $60^{\circ}$ head up tilt and supine posture calculated as $\triangle \mathrm{AVP}$. (D) Heart/mediastinum $(\mathrm{H} / \mathrm{M})$ ratio from ${ }^{123}$-metaiodobenzylguanidine (MIBG) delayed imaging. (E) Systolic blood pressure increase during noradrenaline infusion test. ${ }^{*} \mathrm{p}=0.004,{ }^{* *} \mathrm{p}=0.0003,{ }^{* * *} \mathrm{p}=0.003,{ }^{* * * *} \mathrm{p}=0.002,{ }^{* * * *} \mathrm{p}=0.0004$ ， Mann-Whitney $\mathrm{U}$ test.
Autonomic nervous system testing in PAF and MSA

We found significant differences between PAF and MSA patients with respect to the following:

- orthostatic hypotension evaluated by the head up tilt test (mean (SD): PAF, 68.9 (22.5) mm Hg; MSA, 36.3 (20.4) $\mathrm{mm} \mathrm{Hg} ; \mathrm{p}=0.004$ (fig lA);

- noradrenaline concentration: PAF, 36.1 (23.2) pg/ml; MSA, 189.9 (121.9) pg/ml; p = 0.0003 (fig 1B);

- $\triangle A V P$ : PAF, (10.7) pg/ml; MSA, 0.34 (0.62) pg/ml; $\mathrm{p}=0.003$ (fig $\mathrm{IC})$;

- H/M ratio: PAF, 1.15 (0.05); MSA, 2.04 (0.44); $\mathrm{p}=0.002$ (fig 1D);

- noradrenaline infusion test: PAF, 70.1 (23.2) mm Hg; MSA, 23.7 (11.0) $\mathrm{mm} \mathrm{Hg} ; \mathrm{p}=0.0004$ (fig $\mathrm{lE}$ ).

\section{Clinical course of autonomic failure}

Kaplan-Meier curves depicting the natural clinical course of PAF and MSA are shown in fig 2. Hypohidrosis, faintness and syncope, constipation, urinary dysfunction, and respiratory disturbance were assessed sequentially.

\section{Hypohidrosis}

Six patients noted hypohidrosis or anhidrosis as an initial symptom, and seven became aware of hypohidrosis within five years of onset. Hypohidrosis was one of the earliest and most important symptoms of patients with PAF. In contrast, patients with MSA noted hypohidrosis at a significantly later stage of disease $(p=0.027)$.

\section{Faintness and syncope}

These symptoms represented orthostatic hypotension. Usually faintness preceded syncope. Faintness was often noted as an initial autonomic symptom in PAF. Four of eight patients first noted hypohidrosis in the same year as they first experienced faintness. In our series, five patients complained of faintness as an initial symptom, and seven noted faintness within five years of onset. Syncope appeared at (mean (SD)) 6 (7) years after the onset of faintness, and half the patients had experienced syncope within five years. However, two patients first noted syncope more than 19 years after experiencing faintness. In patients with MSA, faintness was observed later in the course of illness, with risk of progression to syncope differing significantly between the two groups $(\mathrm{p}=0.002)$.

\section{Constipation}

Constipation was among the early symptoms of PAF. In our series, three patients noted constipation as an initial symptom, and five noted constipation within five years of onset; all patients complained of constipation within 13 years. Constipation was the second earliest symptom in our PAF patients, while patients with MSA also complained of constipation at a relatively early stage of disease. No significant differences were seen between the two groups in time from onset of first symptom to development of constipation $(p=0.46)$.

\section{Urinary dysfunction}

In the early stages few PAF patients noted urinary dysfunction, while at a later stage most patients had this complaint. In our series, urinary dysfunction appeared at (mean (SD)) 9 (9) years after the onset of hypohidrosis, faintness, and constipation. Only three patients noted urinary urgency, urinary frequency, or incontinence in the first five years. Among types of urinary dysfunction, difficulty in urination was rare in PAF patients. We evaluated the results of urodynamic studies in five of the eight PAF patients, at four, 

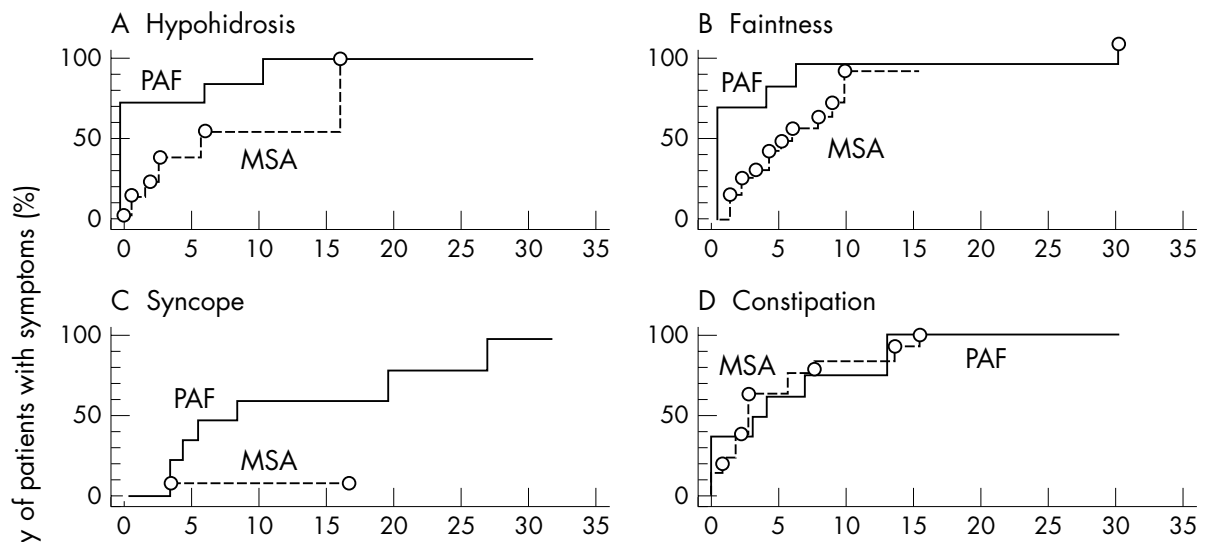

E Urinary dysfunction

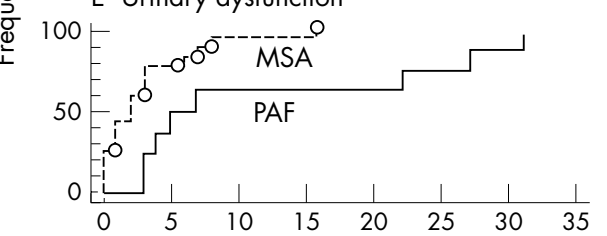

F Respiratory dysfunction

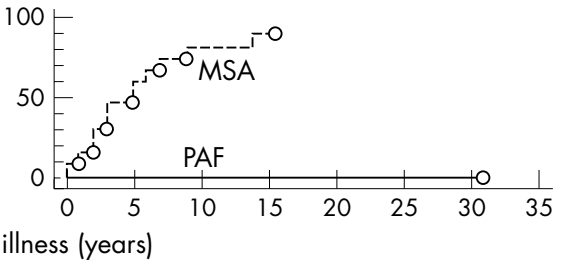

Figure 2 Progression of autonomic symptoms including hypohidrosis (A), faintness (B), syncope (C), constipation (D), urinary dysfunction (E), and respiratory disturbance (F) in patients with pure autonomic failure (PAF) and multiple system atrophy (MSA). Hypohidrosis was an earlier symptom in $P A F$ than in MSA (panel $A, p=0.027$ ). Faintness and syncope were earlier symptoms in PAF than in MSA (panel $B, p=0.04 ; p a n e l=1, p=0.002$ ). Development of constipation was similar between the two diseases (panel D). Urinary dysfunction was a later symptom in PAF than in MSA (panel E, $p=0.004)$. Respiratory disturbance did not occur in our PAF patients, but MSA patients had these problems at an early stage (panel $F, p=0.0004)$.

six, 10, 13, and 17 years after the onset of PAF, respectively. Two of the five patients were essentially asymptomatic and had normal study results. Three patients were symptomatic, one of whom had an overactive bladder and the other an underactive bladder; the third had normal results. In our series, all eight patients had urinary dysfunction by 30 years after onset. Thus urinary dysfunction typically emerged in late stage PAF. In contrast, MSA patients developed urinary dysfunction at a very early stage of their disease $(p=0.004)$, often as an initial autonomic symptom in about a quarter of the patients. Within five years, more than $75 \%$ of MSA patients had urinary dysfunction, especially difficulty in urination. Thus urinary symptoms occurred early and were particularly prominent in MSA.

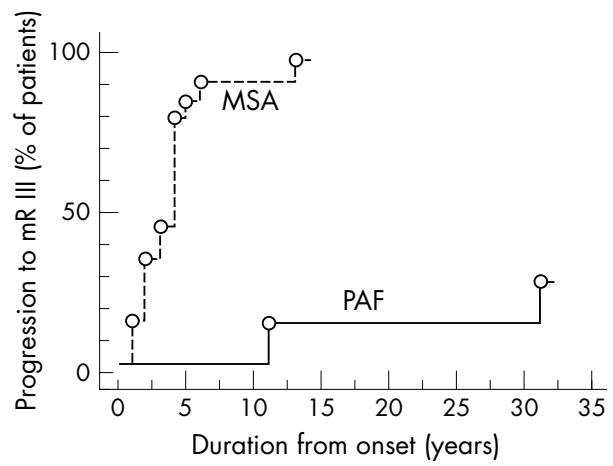

Figure 3 Differences in time remaining independent in activities of daily living (ADL) assessed by the modified Rankin scale between patients with pure autonomic failure (PAF) and multiple system atrophy (MSA). Round symbols represent censored data. Significant differences were seen between PAF and MSA for three ADL milestones and for survival, by Kaplan-Meier analysis and log-rank tests. mR III, modified Rankin scale, grade III (moderate impairment requiring minimal support such as a cane, stair rails, and so on): difference between PAF and MSA significant at $p<0.0001$.

\section{Respiratory disturbances}

Respiratory disturbances such as sleep apnoea were uncommon in patients with PAF. Indeed, in our series, no patient had respiratory difficulties in 30 years of follow up. In contrast, respiratory disturbance was one of the most important features in patients with MSA $(p=0.0004)$. About half the MSA patients had this complaint within five years, and subsequently the prevalence of respiratory disturbances increased. More than $80 \%$ of the MSA patients had respiratory disturbances by 10 years.

\section{Progression of orthostatic hypotension and noradrenaline supersensitivity \\ Orthostatic hypotension}

Orthostatic hypotension (fig 1A) was a major clinical feature in PAF, being marked even in the early stages of the disease. Blood pressure fall varied from 34 to $108 \mathrm{~mm} \mathrm{Hg}$ at presentation to our hospital, and the extent of orthostatic hypotension progressed markedly in most patients over the next two to 11 years. In seven patients blood pressure fell by more than $50 \mathrm{~mm} \mathrm{Hg}$, and most patients experienced syncope.

\section{Noradrenaline supersensitivity}

The noradrenaline infusion test estimates denervation supersensitivity at peripheral noradrenaline receptors, suggesting disease involvement of the peripheral sympathetic nervous system. At an early stage, PAF patients all showed excessive rises in blood pressure of $30 \mathrm{~mm} \mathrm{Hg}$ or more with infusion of a low concentration of noradrenaline ( 3 or $0.3 \mu \mathrm{g} / \mathrm{min}$ ), indicating the presence of denervation supersensitivity (fig 1E). After two to 11 years, however, the extent of blood pressure rise in response to noradrenaline infusion was smaller than at an early stage, suggesting emergence of some compensatory mechanism or secondarily induced insensitivity of noradrenaline receptors. 


\section{Activities of daily living and prognosis}

PAF patients did not show diminishing capacity for activities of daily living (ADL) up to a late stage (fig 3). In our series three patients died, but they maintained nearly normal ADL throughout their lives. One patient who died at 90 years, 12 years after disease onset, could walk alone without assistive devices until he was 89 years old (modified Rankin scale, 0 tol); rapid deterioration in the last year of life resulted from a subdural haematoma. Another patient who died at 82 years, 32 years after onset, could perform all his daily activities unassisted until he was 81 . He was essentially bedridden for the last year of life because of myelodysplastic syndrome. The third patient, who died aged 84 years 12 years after disease onset, remaining healthy and active (modified Rankin scale 0 to 1) until he died suddenly of a severe stroke.

Although both MSA patients and PAF patients have severe autonomic disturbances, functional and survival prognoses ${ }^{16}$ were significantly worse in MSA than in PAF. In our series, median time from onset to modified Rankin scale grade III in MSA was four years $(\mathrm{p}<0.0001 v$ PAF $)$; grade IV, seven years $(p=0.0009)$; grade $V$, nine years $(p<0.0001)$; and death, 11 years $(p=0.003)$. In contrast to MSA, PAF carried a relatively good prognosis for function and survival.

\section{DISCUSSION}

PAF is a chronic progressive neurodegenerative disease characterised by severe autonomic failure without other neurological deficits. Uniquely, PAF patients can maintain a long healthy life, in contrast to patients with other types of primary autonomic failure. Pathological reports of PAF have described Lewy bodies in the intermediolateral grey columns of the thoracolumbar spinal cord, suggesting that PAF is a form of Lewy body disease..$^{3-30}$

Our study is the first assessment of long term progression of autonomic symptoms and ADL status in PAF, particularly in comparison with MSA. Although a consensus has been reached over the diagnostic criteria for $\mathrm{PAF},{ }^{8}$ long term follow up observation of the clinical features is important to identify the differences between PAF and autonomic failure in other neurodegenerative diseases, particularly MSA and Parkinson's disease with autonomic failure..$^{7812}$ We investigated clinical features of eight patients with PAF over follow up periods ranging from seven to 32 years.

It is generally accepted that patients with PAF have autonomic failure resulting in peripheral but not central involvement. The results of supine noradrenaline levels, $\triangle \mathrm{AVP},{ }^{123} \mathrm{I}-\mathrm{MIBG}$, and the noradrenaline infusion test clearly confirm this. In contrast, patients with MSA have patterns suggesting a predominantly central involvement, although some patients with probable MSA also have low noradrenaline concentrations, increased $\triangle \mathrm{AVP}$, a reduced $\mathrm{H} / \mathrm{M}$ ratio, and raised blood pressure during the noradrenaline infusion test. These neuropharmacological tests would be useful for differentiating PAF from MSA early in the course of the illness. Further studies are needed to clarify their sensitivity, specificity, and positive predictive value.

In our study, orthostatic hypotension and related faintness and syncope were the most important clinical features of PAF, and developed at a very early stage. Furthermore, orthostatic hypotension worsened gradually as the disease progressed in spite of medical treatment for hypotension. In contrast, MSA patients were less likely to have syncope than PAF patients. Progression of MSA is relatively rapid, ${ }^{16}$ so MSA patients are often wheelchair bound or nearly bedridden before showing severe hypotension with syncope. ${ }^{16}$ About half the patients with MSA noted faintness by four years after onset, at a time when most of them were wheelchair bound and spent a considerable amount of their waking time lying down. This may limit the exposure of MSA patients to syncope.

Another important autonomic abnormality observed in PAF was sudomotor impairment. Hypohidrosis or anhidrosis was a major complaint in patients with PAF. Emergence of orthostatic hypotension, sometimes with loss of consciousness, and sudomotor dysfunction at a very early stage were striking characteristic features in PAF, in contrast to MSA where these symptoms were absent in the early phase of the disease.

A striking clinical characteristic of PAF was the absence of respiratory dysfunction such as sleep apnoea until a very late phase of disease. This feature again contrasted with MSA, where respiratory dysfunction was a major problem, threatening life in the later phase of disease.

Constipation and urinary dysfunction are among the characteristic symptoms of primary autonomic failure syndrome including PAF, MSA, and Parkinson's disease with autonomic failure. ${ }^{30} 31$ Urinary problems have been documented in the past to some extent, ${ }^{9} 11{ }^{12}$ representing a characteristic feature of PAF, especially in the late phase. Sakakibara et $a l^{32}$ reported that all six of their patients with PAF who complained of urinary disturbances showed abnormalities on urodynamic studies. In our series, five of eight patients underwent urodynamic evaluation, and two with urinary symptoms showed a hyperactive or underactive bladder. However, the severity of the urodynamic abnormalities and associated symptoms was mild, in agreement with the previous report. ${ }^{32}$ In contrast, patients with MSA have severe urinary dysfunction, especially difficulty in urination ${ }^{33}$ and nocturnal urinary frequency, with residual urine, detrusor hyperreflexia, low compliance, and detrusor sphincter dyssynergy on urodynamic studies. Intermittent self catheterisation is often required even early in the course of the illness.

On the basis of these observations, we can assume that orthostatic hypotension and sudomotor dysfunction precede urinary dysfunction and particularly respiratory dysfunction in the development of autonomic disturbances in PAF, while in MSA urinary dysfunction precedes orthostatic hypotension and sudomotor dysfunction, and respiratory dysfunction is a serious problem even at an early stage. Modes of progression of autonomic symptoms seem to be an important way of distinguishing between PAF and MSA.

The evolution of the change in blood pressure during the noradrenaline infusion test in PAF is difficult to explain. While the clinical features became worse over the course of several years in PAF patients, in contrast the degree of blood pressure elevation during the test became smaller with time. The same method was used for the test on each occasion, and no previous reports provide an explanation for this phenomenon. Age related changes such as atherosclerosis or changes in drug treatment might have contributed, but further study is necessary.

Patients with PAF had a better prognosis than those with MSA. Even the three patients with PAF who died during follow up lived independently until one or two years before they died all died of concurrent diseases. Various factors contributed to this advantage in ADL and long term prognosis. First, patients with PAF did not have severe urinary disturbances, which would lessen the risk of recurrent urinary infections, and they also did not have life threatening respiratory failure. Second, while management of orthostatic hypotension remains challenging late in the course of illness, administration of plasma volume expansion fluids, fludrocortisone, and sympathomimetic agents can be effective in ameliorating symptoms and preventing faintness and syncope with resulting head injuries or bone fractures which could compromise ADL and survival. Third, patients 
with PAF in this study showed no motor or cognitive impairment. No parkinsonism or dementia, which would have affected daily activities or required additional treatment, was evident during the course of their illness. Further studies are needed to evaluate the significance of the pathological background for temporal features of autonomic, motor, and cognitive involvements.

The precise epidemiology of PAF has not been assessed, either in Japan or in Western countries. To our knowledge, relatively few cases of PAF have been studied or described, and necropsy reports are far less common than for MSA. In our Japanese series, more than 200 patients with MSA were referred to hospital during the course of the study, but only eight patients with PAF were diagnosed during the same period. Although physician referral patterns may have on influence, PAF appears to be uncommon in Japan compared with MSA. Further studies should be undertaken to clarify the incidence and prevalence of PAF worldwide.

\section{Authors' affiliations}

N Mabuchi, M Hirayama, H Watanabe, H lto, K Hamada, G Sobue, Department of Neurology, Nagoya University Graduate School of Medicine, Nagoya, Japan

Y Koike, Department of Health Science, Nagoya University Graduate School of Medicine

R Kobayashi, Department of Neurology, National Nagoya Hospital

Competing interests: none declared

\section{REFERENCES}

1 Rosecan M, Glaser RJ, Goldman ML. Orthostatic hypotension, anhidrosis, and impotence. Circulation 1952:6:30-40.

2 Drenick EJ. Orthostatic hypotension in the presence of hypertensive cardiovascular disease. Ann Intern Med 1957;47:124-31

3 Roessmann U, van der Noort S, McFarland DE. Idiopathic orthostatic hypotension. Arch Neurol 1971;24:503-10.

4 Ochiai J, Kobayashi T, Gotou I, et al. Clinical study of two cases of "pure progressive autonomic failure" ${ }^{\prime \prime}$. Shinkei Naika 1986;24:297-9.

5 Tsuboi K, Nakano K, Tokuhisa H, et al. A case of slowly progressive idiopathic orthostatic hypotension. Junkan Naika 1983;3:1078-86.

6 Bradbury S, Eggleston C. Postural hypotension. A report of three cases. Am Heart J 1925; 1:73-86.

7 Bannister R, Mathias C, Polinsky R. Autonomic failure. In: A textbook of clinical disorders of the autonomic nervous system, 2nd ed. Oxford: Oxford University Press, 1988:267-88.

8 Consensus committee. The consensus committee of the American Autonomic Society and the American Academy of Neurology. Consensus statement on the definition of orthostatic hypotension, pure autonomic failure, and multiple system atrophy. Neurology 1996;46:1470.

9 Tamura N, Shimazu K, Yamamoto T, et al. Clinical features and nosology of pure autonomic failure. Jiritsu Sinkei 1995;32:435-42.

10 Akimitsu T, Maeda T, Hara M, et al. Pure progressive autonomic failure presenting severe orthostatic hypotension. Intern Med 1993;32:122-7.
11 Asahina M Hattori T. Pure autonomic failure: differential diagnosis and limitations of treatment. Shinkei Naika 2002;57:29-34.

12 Davidson C, Morgan DB. Long survival in orthostatic hypotension. Case report and a review of the literature. J Chron Dis 1976;29:733-42.

13 Brigden W. Postural hypotension. Br Heart J 1946;8:103-9

14 Kaufmann H. Primary autonomic failure: Three clinical presentation of one disease? Ann Intern Med 2000;133:382-3.

15 Bannister R, Mathias CJ. Autonomic failure. In: A textbook of clinical disorders of the autonomic nervous system, 4th ed. Oxford: Oxford University Press, 1999:307-16.

16 Watanabe H, Saito $Y$, Terao S, et al. Progression and prognosis in multiple system atrophy. An analysis of 230 Japanese patients. Brain 2002:125:1070-83.

17 Gilman S, Low PA, Quinn N, et al. Consensus statement on the diagnosis of multiple system atrophy. J Neurol Sci 1999;163:94-8.

18 Hirayama M, Koike Y. Physiological test for autonomic nervous system. Nippon Rinsho 1997:714:487-90.

19 Hirayama M, Koike Y. Pharmacological test for autonomic nervous system. Nippon Rinsho 1997;714:491-5.

20 Watanabe $\mathrm{H}$, leda T, Katayama T, et al. Cardiac ${ }^{123} \mathrm{I}$-metaiodebenzylguanidine (MIBG) uptake in dementia with Lewy bodies: comparison with Alzheimer's disease. I Neurol Neurosurg Psychiatry $2001 ; 70: 781-3$.

21 Hamada K, Hirayama M, Watanabe $H$, et al. Onset age and severity of motor impairment are associated with reduction of myocardial ${ }^{123}$ I MIBG uptake in Parkinson's disease. J Neurol Neurosug Psychiatry 2002;74:423-6.

22 Hirayama M, Hakusui S, Koike $Y$, et al. A scintigraphical qualitative analysis of peripheral vascular sympathetic function with meta$\left[{ }^{123} \mid\right]$ iodobenzylguanidine in neurological patients with autonomic failure. J Auton Nerv Syst 1995;53:230-4.

23 van Ingelghem $E$, van Zandijcke $M$, Lammens $M$, et al. Pure autonomic failure: a new case with clinical, biochemical, and necropsy data. J Neurol Neurosurg Psychiatry 1994;57:745-7

24 Hague K, Lento P, Morgello S, et al. The distribution of Lewy bodies in pure autonomic failure: autopsy findings and review of the literatures. Acta Neuropathol (Berl) 1997:94:192-6.

25 Hishikawa N, Hashizume Y, Hirayama M, et al. Brainstem-type Lewy body disease presenting with progressive autonomic failure and lethargy. Clin Auton Res 2000;10:139-43.

26 Arai K, Kato N, Kashiwado K, et al. Pure autonomic failure in association with human $\alpha$-synucleinopathy. Neurosci Lett 2000;296:171-3.

27 Miura H, Tsuchiya K, Kubodera T, et al. An autopsy case of pure autonomic failure with pathological features of Parkinson's disease. Rinsho Shinkeigaku 2001;41:40-4.

28 Johnson RH, Lee de JG, Oppenheimer DR, et al. Autonomic failure with orthostatic hypotension due to intermediolateral column degeneration. Q J Med 1965;138:276-9.

29 Terao Y, Takeda K, Sakuta M, et al. Pure progressive failure: a clinicopathological study. Eur Neurol 1993;33:409-15.

30 Noda K, Katayama S, Watanabe C, et al. Pure autonomic failure with motor neuron disease: Report of a clinical study and postmortem examination of a patient. J Neurol Neurosug Psychiatry 1994;57:745-7.

31 Niimi $Y$, leda $T$, Hirayama $M$, et al. Clinical and physiological characteristics of autonomic failure with Parkinson's disease. Clin Auton Res 1999;9:139-44.

32 Sakakibara R, Hattori T, Uchiyama T, et al. Micturitional disturbance in pure autonomic failure. Neurology 2000;54:499-501.

33 Sakakibara R, Hattori T, Uchiyama T, et al. Urinary dysfunction and orthostatic hypertension in multiple system atrophy: which is the more common and earlier manifestation? J Neurol Neurosurg Psychiatry 2000;68:65-9 